\title{
Analysis of early postoperative outcomes of combined simultaneous and multistage surgeries
}

Alymkadyr S. Beyshenaliev ${ }^{1}$, Nurgazy M. Zhumagulov², Taalaibek K. Atabaev², Begmamat M. Nyshanov²

${ }^{1}$ Propaedeutic surgery department of KSMA named after I.K.Akhunbaev, Bishkek, Kyrgyzstan

${ }^{2}$ Osh Interregional United Clinical Hospital, Osh, Kyrgyzstan

\begin{abstract}
:
Objective: comparative analysis of the postoperative outcome of combined surgery for concomitant abdominal and pelvic (gynecological) pathology

Methods: Overall, 346 surgical patients with concomitant abdominal and gynecological surgical diseases were analyzed retrospectively in postoperative period.

Results: In the early postoperative period complications developed in groups of extreme high and high operationalanesthetic risk (ASA III and ASA IV).

Conclusion: According to results of our study, combined surgical interventions especially those performed from a single surgical approach, slightly increase the severity of the operation compared to isolated interventions performed for the same underlying diseases.
\end{abstract}

Key words: combined operations, concomitant surgical diseases, operative-anesthetic risk, scope of operation, postoperative complications, abdominal surgery, gynecological operations.

(Heart Vess Transplant 2019; 3: doi: 10.24969/hvt.2019.171)

\section{Introduction}

The contemporary achievements of surgery and anesthesiology enhanced the feasibility of combined operations as extensive and complex interventions (114). According to literature review, proportion of patients with two or three concomitant surgical pathology counts for $20-30 \%$ and possess a tendency to increase $(2,7,12)$. Despite the wide variety of trials devoted to etiological factors of combined surgical diseases, few investigations on operative management options exist and vast majority of these studies are controversial $(2,9)$.

The management of concomitant surgical diseases requires the solution of two consequent issues: which method is preferred in accordance with preoperative risk assessment: multistage or simultaneous tactics and after the decision-making establishing an appropriate time for carrying out of a chosen intervention $(3,10)$.

According to our retrospective analysis, the simultaneous interventions are implemented in clinical practice extremely rarely, which do not fulfill their real requirements. This issue is explained by several reasons: incomplete examination of patients in the preoperative period, insufficient intraoperative revision of the abdominal and pelvic cavities, overstatement of the operational risk when determining the possibility of performing simultaneous operations, the habitude of surgeons to perform multistage treatment of combined diseases, increased responsibility of the surgeon in case of possible unsuccessful outcome, technical and psychological unpreparedness of surgeons, gynecologists and anesthesiologists for extensive intervention $(4,5,13)$.

Address for Correspondence: Beyshenaliev A.S., Propaedeutic surgery department of KSMA named after I.K.Akhunbaev, Bishkek, Kyrgyzstan

Received: 16.11.2018 Revised: 02.03.2019 Accepted: 15.11.2019

Copyright @ 2019 Heart, Vessels and Transplantation 
Surgical treatment of patients with combined pathology requires a specific approach, both in preoperative preparation and in intra- and postoperative management. In advance determination of operational scope and arrangement of the operating team for extensive intervention depend on detailed preoperative evaluation of patients. Thus, consultation of gynecologist and risk assessment of anesthesiologist are obligatory before planning the operation strategies and scope of intervention.

The operational access has a leading role in a combined emergency surgery, because of its contribution in defining the extension of operation and appropriate patient selection. The extremely important point in decision-making of simultaneous operation is that, the risk of intervention should not exceed the risk of the disease itself.

The development of postoperative complications and their physical assessment remain challenging in daily practice of surgery $(5,7,8)$. Currently the combined operations are frequently associated with fatal complications $(2,6,12)$.

The challenge of objectivity is found frequently in operational risk assessment, as long as the doctors do not evaluate the risk parameters equally. Often the necessary preoperative assessment is carried out by individual opinion.

From the past, ongoing several stratification tools are proposed for ratification of operational and postoperative risk assessment. A number of published papers were devoted to the operational risk assessment depending on the character of surgical intervention and the preoperative status of the patient $(3,7)$.

We understand the operative risk as the degree of presumed risk for patient during surgical operation and anesthesia.

The workup of unified criteria for prognosis of complications and mortality in case of combined surgical pathology remains challenging due to irresistible contradictions of two or three incomparable pathologies and enormous difficulties in combined surgery.

The aim of our study is analysis of surgical outcomes of 123 patients with combined surgical and gynecological diseases depending on the operational and anesthetic risk.

\section{Methods}

\section{Study population}

We conducted retrospective study from January of 2014 to December of 2017 in surgical department of Osh Interregional United Clinical Hospital. Overall, 346 female patients with combined surgical and gynecological conditions were enrolled into study. The main group consisted of 123 women underwent simultaneous operations, the control group had 223 patients underwent multistage surgery of abdominal and pelvic organs.

Criteria for inclusion of patients to the study were patients with a gallstones, appendicitis and abdominal hernia with combined gynecological pathology of pelvic organs diagnosed pre- and intraoperatively.

All patients were carefully examined in preoperative workup, investigations included thorough history taking, physical assessment, standard laboratory tests, abdominal and pelvic ultrasound examinations, consultations of specialists in case of concomitant diseases.

In general, the groups were statistically comparable in age and frequency of comorbidities.

\section{Surgical approaches}

Operations in both groups were performed by generally accepted conventional methods.

Access to the hepatobiliary organs was carried out in the right hypochondrium by an oblique or transverse incision of the skin, subcutaneous tissues, superficial fascia, right rectal abdominal muscle and partial incision of right abdominal flat muscles, intraabdominal fascia and peritoneum. Peritoneum incisions were isolated with sterile towels. 
Appendectomy was performed through McBurney (Volkovich-Dyakanov's) approach (15). Hernia repair was performed by generally accepted accesses depending on localization.

Access to the genitalia was carried out according to Pfannenstiel by cross dissection over the skin of suprapubic zone, subcutaneous tissue, superficial fascia, anterior duplicates of vagina of abdominal rectal muscles, partial incision of aponeuroses of the external oblique muscles and subsequent longitudinal dissection from the umbilicus to the pubic symphisis via the linea alba, and eventual opening of visceral fascia and peritoneum.

\section{Operative-anesthetic risk assessment}

Risk is evaluated according to ASA physical status assessment system (16).

\section{Statistical analysis}

We used descriptive statistics to present data.

\section{Results and discussion}

\section{Patients' population}

The 18 female patients were observed at the age of 21 to 30 years (14.6\%), 29 patients from 31 to 40 years (23.6\%), and the most numerous group included people aged 41 to 50 years old-42 patients (34.2\%). Patients aged from 51 to 60 years were $32(26.0 \%)$, and over the 60 years - 2 patients (1.6\%) (Fig. 1).

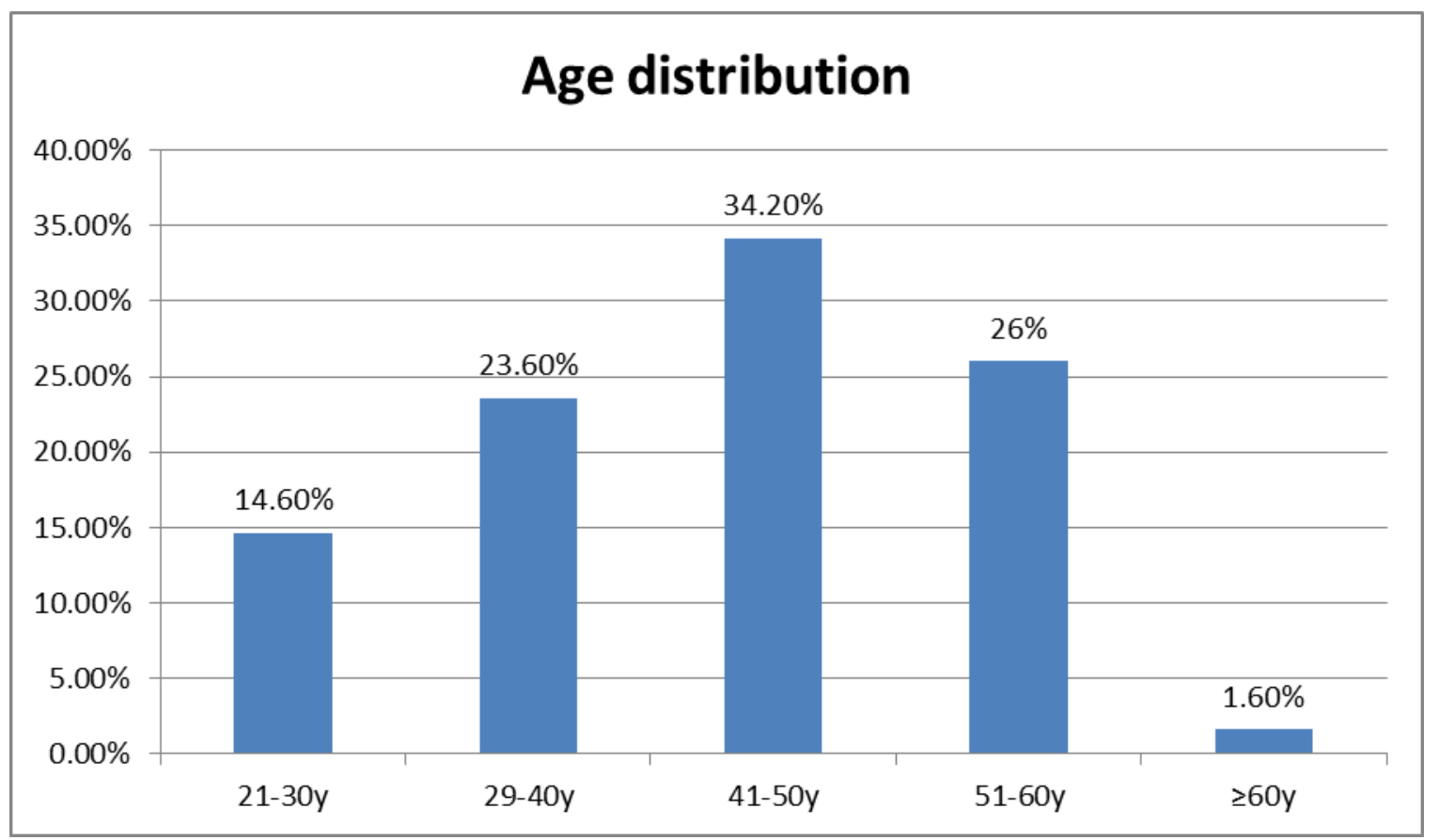

Figure 1. Distribution of patients according to age.

The 14 patients (11.4\%) had concomitant cardiovascular and respiratory diseases, which required through preoperative preparation.
Distribution of patients according to character of combined surgical and gynecological diseases is presented in Table 1 and Figure 2. 


\begin{tabular}{|l|c|c|}
\hline \multirow{2}{*}{ Table 1. Distribution of patients by pathology type } & \multicolumn{2}{|c|}{ Total } \\
\cline { 2 - 3 } & Absolute number & $\%$ \\
\hline Cholecystitis + uterine fibromyoma & 26 & 21.1 \\
\hline Cholecystitis + ovarian cyst & 7 & 5.7 \\
\hline Cholecystitis + uterine fibromyoma + ovarian cyst & 8 & 6.5 \\
\hline Acute appendicitis + uterine fibromyoma & 7 & 5.7 \\
\hline Acute appendicitis + uterine fibromyoma + ovarian cyst & 1 & 0.8 \\
\hline Acute appendicitis +ovarian apoplexy & 17 & 13.8 \\
\hline Acute appendicitis + ovarian cyst & 18 & 14.6 \\
\hline Hernia+ uterine fibromyoma & 21 & 17.2 \\
\hline & 10 & 8.1 \\
Hernia+ uterine fibromyoma+ ovarian cyst & & \\
\hline Hernia+ovarian cyst & 8 & 6.5 \\
\hline All & 123 & 100 \\
\hline
\end{tabular}

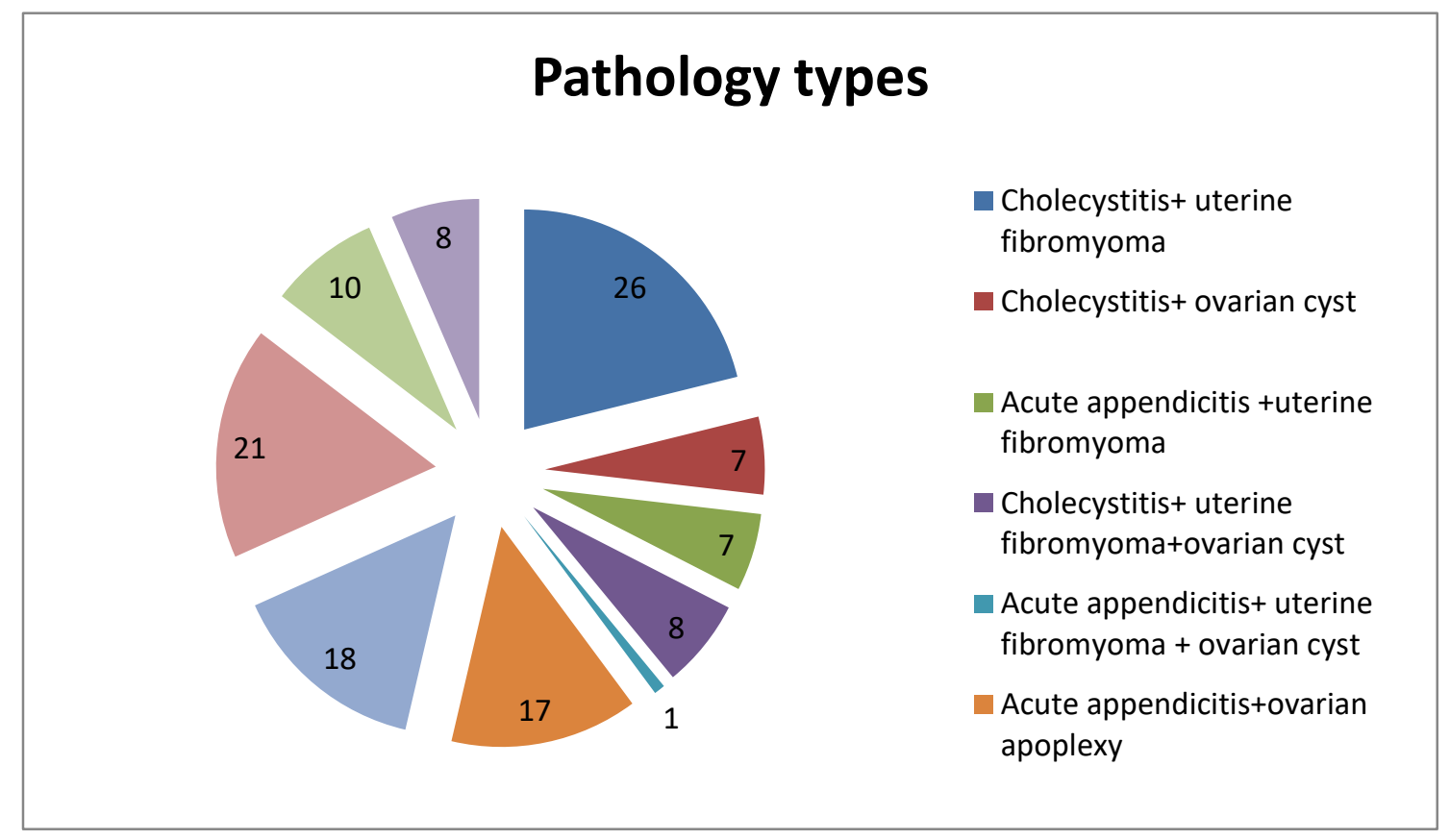

Figure 2. Patients` distribution according to pathology type

The patients with appendicitis prevailed among combined pathology. As seen from the Table 1, gallbladder surgery was implemented in 41 cases (33.3\%), in combination with uterine fibromyoma - 26, with an ovarian cyst- 7 , and in combination with uterine fibromyoma and ovarian cyst in 8 patients. The $43(35.0 \%)$ patients were operated for acute appendicitis, in combination with uterine fibroids-7, uterine fibroids + ovarian cyst -1 , ovarian apoplexy and ovarian rupture -17 . Among patients $39(31.7 \%)$ were operated due to abdominal hernia, and in combination with uterine fibroids -21 , uterine fibromyoma + ovarian cyst -10, and ovarian cyst -8 . 
Additionally, we investigated the patients who had undergone isolated operations for surgical and gynecological diseases. These patients composed a control (comparative) group, which included 223 patients with gynecologic 114 and surgical 109 conditions. Volume of isolated operations varied from supravaginal hysterectomy, removal of appendages, cholecystectomy, appendectomy, ventroplasty.
In the control group (Fig. 3), patients were operated for uterine fibroids in $71(62.3 \%)$ cases, whereas 43 (37.7\%) patients underwent ovarian cyst surgery. The $85(78.0 \%)$ patients of control group were exposed to cholecystectomy, 13 (11.9\%) patients underwent appendectomy, and 11 (10.1\%) patients were operated for hernia of the anterior abdominal wall.
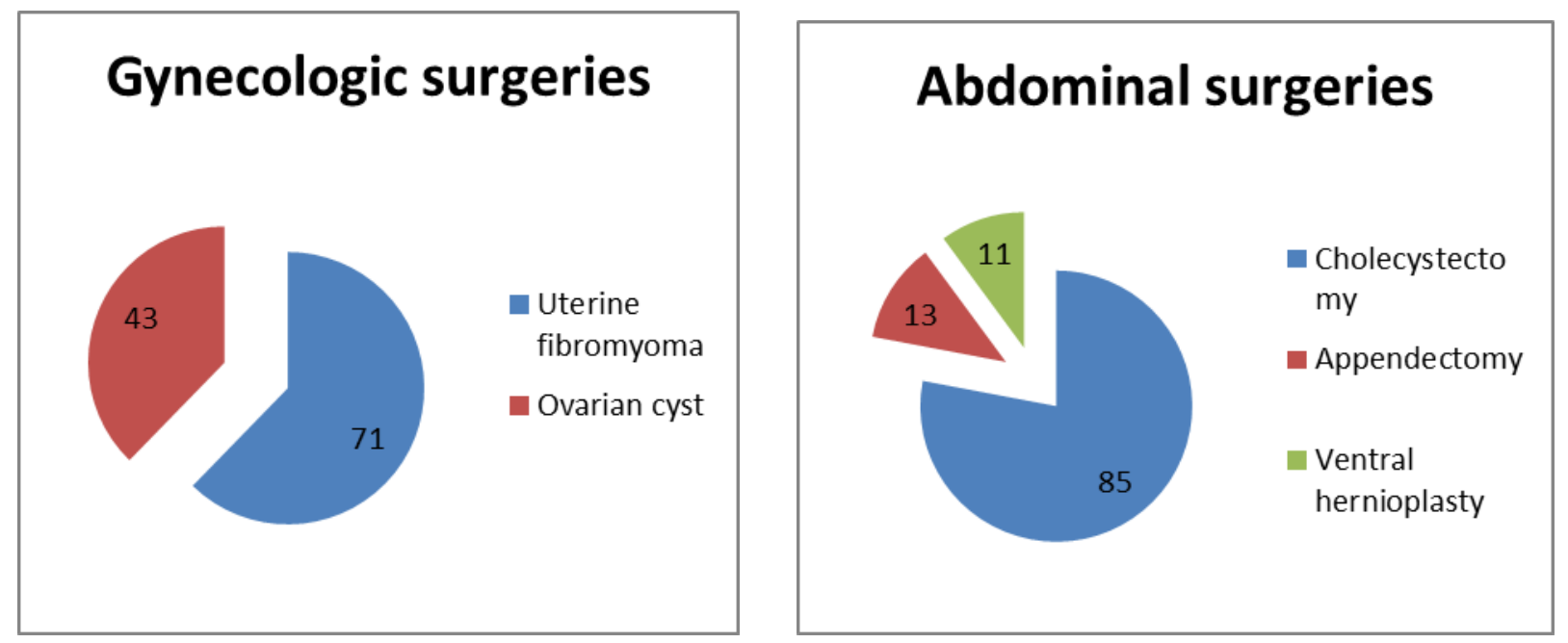

Figure 3. Types of surgeries

\begin{tabular}{|l|l|l|}
\hline \multirow{3}{*}{ Table 2. The degree of operative - anesthetic risk of simultaneous surgery } \\
\cline { 2 - 3 } & Number of patients \\
\cline { 2 - 3 } & Absolute number & With complications, $\mathbf{n}$ (\%) \\
\hline ASA I & 31 & - \\
\hline ASA II & 79 & $3(3.8)$ \\
\hline ASA III & 11 & $5(45.5)$ \\
\hline ASA IV & 2 & $2(100)$ \\
\hline Total & $\mathbf{1 2 3}$ & $\mathbf{1 0 ( 8 . 1 )}$ \\
\hline
\end{tabular}

The complications developed in 10 (8.1\%) cases among 123 patients encountered combined surgery (Table 2). All complications were associated with the main stage of simultaneous operation.

The developed complication types in early postoperative period were: pneumonia registered in two $(1.6 \%)$, bile leakage in one $(0.8 \%)$, partial adhesive intestinal obstruction in one $(0.8 \%)$, postoperative wound suppuration in six (4.9\%) patients. Mortality was not observed in early postoperative period.

In the control group, earlier postoperative complications were observed in 16 (7.1\%) cases.

Postoperative complications most commonly were registered in patients with older and advanced age. This is largely due to the prevalence of elder ones among the patients underwent combined operations. In addition to combined surgical diseases, patients of advanced age had cardiovascular and respiratory disorders. Distribution of complications according to operative-anesthetic risk in simultaneous surgery (Table 2) demonstrated no complications in group with ASA I, 3.8\% of patients developed complications in group of ASA II, 45.5\% - ASA III and 100\% - ASA IV.

\section{Study limitations}

Our study is not directed to evaluate a certain preoperational parameter of risk assessment, such as advanced age, cardiovascular comorbidity or parity status of gynecological patient. 
In further works, we are planning to concentrate on analysis of postoperative complications depending on certain parameter.

In this study, only open surgeries are included, but in next surveys, we also take in account endoscopic procedures.

\section{Conclusion}

According to the retrospective analysis and the study of prognostic indicators, we concluded that, combined operations are contraindicated for patients with high and extremely high degree of operative-anesthetic risk; therefore, they should be planned to multi-stage operations. All postoperative complications emerged due to underlying disease, advanced age and presence of cardiovascular and respiratory comorbidities. Despite the development of various postoperative complications and moderately increased effort for the surgical team, combined operations must be implemented in clinical practice according to feasibility of appropriate conditions: patient commitment, well-trained and psychologically prepared surgeon, complete preoperative investigations, adequate operative-anesthetic risk assessment, and sufficient revision of cavities.

According to results of our study, combined surgical interventions especially those performed from a single surgical approach, slightly increase the severity of the operation compared to isolated interventions performed for the same underlying diseases.

Simultaneous operations are not associated with severe operational complications, which could be an expected feature of solely this type of operation.

Peer-review: Internal and external

Conflict of interest: None to declare

Authorship: A.S.B., N.M.Zh., T.K.A. and B.M.N equally contributed to study and preparation of manuscript Acknowledgement and funding: None to declare

\section{References}

1.Adamyan, LV. Combined operations in gynecology: issues of classification and methodological approach. In: Adamyan LV, Gaidarova AKh, Panin AV, eds. Laparoscopy and hysteroscopy in obstetrics and gynecology. Moscow: 2002. pp. 422-8.

2.Baulina NV, Baulin EA. Simultaneous operations in surgery and gynecology. Journal of Surgery II Grekova. 2004; 163: 87-91.

3.Bogdanov YuV., Tkachuk EN, Bogdanov DYu. Results of simultaneous operations. Endoscopic surgery 2005; 3: $10-2$.

4.Vertkina NV, Khamitov FF. Clinical and economic aspects of simultaneous operations in patients of elderly and senile age. Clinical gerontology 2008; 4: 5 -10 .

5.Zholobova MN. Simultaneous operations in gynecology and surgery: Journal of Obstetrics and Women's Diseases 2005; 4: $11-6$.

6.Ivanov YuV. Modern ideas about combined surgical pathology and simultaneous operations. Annals of Surgery 2007; 4: $17-20$.

7.Makhovskiy VZ, Akseenko VA, Makhovskiy VV. Simultaneous combined operations in emergency surgery and gynecology: Surgery 2008: 9: 41 - 45.

8.Strizheletsky VV. Economic efficiency of simultaneous operations in surgery and gynecology: Moscow Surgical Journal 2008; 1: 26 - 30.

9.Cuschieri A, Dubois F, Mouiel J, Mouret P. Becker H, Buess G, et al. The European experiences with laparoscopic cholecystectomy Am J Surg 1991; 161: 36 -44 .

10.Filiadis I, Hastazeris K, Tsimaris I, Papadopoulos A, Kakoulidis S, Stavropoulos NE. Simultaneous adenomectomy and preperitoneal repair of inguinal hernias by a single incision with the application of polypropylene mesh. Int Urol Nephrol 2003; 35: 19 24.

11.Griffin, S, Abbassi N, Parveen Z, Zafar A, Ali J. Combined abdominal hysterectomy, cholecystectomy and appendectomy: a study of 25 cases in Abbottabad. J Ayub Med Coll Abbottabad 2006; 18: $57-9$.

12. Hart S. Laparoendoscopic single - site combined cholecystectomy and hysterectomy. J Minim Inv Gynecol 2010; 17: 798 - 801.

13.Jocko JA, Shenassa $H$, Singh SS. The role of appendectomy in gynaecologic surgery: a Canadian retrospective case series: J Obstet Gynecol Can 2013; 35: $44-8$.

14. Olivar N. Simultaneous laparoscopic cholecystectomy and laparoscopically assisted vaginal hysteroadnexectomy: Dept. of G. Surg., Hospital of Lodi. Italy. 2007.

15. Olsen DO. Laparoscopic appendectomy. A review. USSC Connecticut 1992: 1- 4.

16. American College of Surgeons' guidelines for care of pediatric surgical patients. J Am Coll Surg 2014; 218: 479-80. 